\title{
Rheological study of structural transitions in triblock copolymers in a liquid crystal solvent
}

\author{
Michael D. Kempe, $\uparrow$ Rafael Verduzco, $\uparrow$ Neal R. Scruggs $\dagger$ and Julia A. Kornfield* \\ Received 12th January 2006, Accepted 23rd March 2006 \\ First published as an Advance Article on the web 3rd April 2006 \\ DOI: $10.1039 / \mathrm{b} 600483 \mathrm{k}$
}

Rheological properties of triblock copolymers dissolved in a nematic liquid crystal (LC) solvent demonstrate that their microphase separated structure is heavily influenced by changes in LC order. Nematic gels were created by swelling a well-defined, high molecular weight ABA block copolymer with the small-molecule nematic LC solvent 4-pentyl-4'-cyanobiphenyl (5CB). The " $\mathrm{B}$ " midblock is a side-group liquid crystal polymer (SGLCP) designed to be soluble in 5CB and the "A" endblocks are polystyrene, which is LC-phobic and microphase separates to produce a physically cross-linked, thermoreversible, macroscopic polymer network. At sufficiently low polymer concentration a plateau modulus in the nematic phase, characteristic of a gel, abruptly transitions to terminal behavior when the gel is heated into its isotropic phase. In more concentrated gels, endblock aggregates persist into the isotopic phase. Dramatic changes in network structure are observed over small temperature windows (as little as $1{ }^{\circ} \mathrm{C}$ ) due to tccche rapidly changing LC order near the isotropization point. The discontinuous change in solvent quality produces an abrupt change in viscoelastic properties for three polymers having different pendant mesogenic groups and matched block lengths.

\section{Introduction}

Block copolymers (BCPs) are a class of soft materials that have been extensively studied in recent decades because they are a versatile system for engineering nanotechnologies, as reviewed by Park, Yoon, and Thomas. ${ }^{1}$ Selection of the length, chemical structure, and connectivity of the component block gives access to a wide variety of morphologies, having sizes typically on the order of $\sim 10-100 \mathrm{~nm}$, which can be ordered with respect to one another in grains of a micrometer or larger., When one block of a BCP is a liquid crystal (LC) polymer, the resulting material exhibits order over a variety of length scales from a few nanometers (the cooperative alignment of LC molecules) up to the larger-scale structure of the $\mathrm{BCP}^{2}$ In these systems the orientational order of the mesophase couples strongly to the microphase separated BCP morphology, and the alignment of the $\mathrm{LC}$ moieties often dictates the orientation of the microdomains. In contrast to the alignment tendency of cylindrical microdomains of a triblock copolymer that normally align in the direction of shear, an orientation perpendicular to the shear plane has been observed when one block is a LC polymer because of the anchoring of the mesogens to the cylinder walls. ${ }^{3}$ The coupling between mesophase order of the LC component and the segregated microstructure of the BCP is also manifested in morphological transitions that either change the microphase separated structure (order-order transition), ${ }^{4,5}$ or trigger the orderdisorder transition coincident with the isotropization temperature of the LC block. ${ }^{6-8}$

Division of Chemistry and Chemical Engineering, California Institute of Technology, 1200 East California Blvd., Pasadena, California, 91125. E-mail: jak@cheme.caltech.edu

$\dagger$ M. D. K., R. V. and N. R. S. contributed equally to this work.
Though conventional BCPs are heavily influenced by mechanical fields, achieving single-crystal alignment of $\mathrm{BCP}$ microstructure with electric fields is difficult, requiring long processing times of up to several hundred minutes under fields

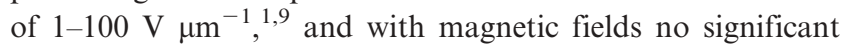
alignment will be obtained even with fields as large as $10 \mathrm{~T}$. The addition of an LCP blocks having anisotropic dielectric and diamagnetic constants increases their responsiveness to electromagnetic fields relative to conventional BCPs. ${ }^{7,10}$ When compared to small molecule liquid crystals, however, the LC-coil polymers have sluggish responses that are relatively weak. Because of the diverse applications of electro-optically active materials, it is desirable to improve the responsiveness of LC-BCPs to electric fields while maintaining the interplay between LC order and BCP microstructure.

We have recently demonstrated ${ }^{11}$ a method for swelling LC-BCPs with small-molecule LC solvent to create LC gels with as little as 5\% polymer content. LC gels self-assembled from LC-BCPs are markedly different from gels made by swelling BCPs with isotropic solvent. In contrast to isotropic solvents, in which solvent quality changes gradually with temperature, there is a discontinuous change in solvent quality in the LC solvent coincident with transitions between LC phases. In turn, the jump in solvent quality produces a sharp phase transition in the BCP solution over a small temperature window of only a few degrees. Furthermore, the birefringence, dielectric anisotropy, diamagnetic anisotropy, and orientational elasticity inherent to the LC solvent give rise to electrooptic and mechano-optic phenomena in LC gels that are forbidden by symmetry in isotropic systems. ${ }^{12}$

The LC gels are formed by dissolving an ABA triblock copolymer having polystyrene (PS) endblocks ("A") and a very long side-group liquid crystalline polymer (SGLCP) 
midblock ('B") in 4-pentyl-4'-cyanobiphenyl (5CB), a wellcharacterized nematic LC. ${ }^{13,14}$ The SGLCP midblock is designed to be soluble in both the isotropic and nematic phases of the solvent. Unfavorable interactions between the PS endblocks and nematic solvent cause segregation into PS-rich domains that function as the physical crosslinks of a spacefilling polymer network.

We report the rheology of LC gels over a range of temperatures and polymer concentrations. Rheology shows differences between LC gels and bulk LC-BCPs brought about by the change in dominant interaction from polymer-polymer to polymer-solvent. In particular, microphase separation of endblocks is coupled to the rapidly changing LC order near the nematic to isotropic phase transition which results in very large changes in the viscoelastic response of the gels.

\section{Experimental}

\section{Gel permeation chromatography (GPC)}

GPC was carried out using three different systems, two analytical and one preparative. The first one used two $30 \mathrm{~cm}$ long PLgel $5 \mu \mathrm{m}$ mixed-C columns from Polymer Laboratories (200 to 2,000,000 $\mathrm{g} \mathrm{mol}^{-1}$ ) connected in series with a DAWN EOS multi-angle laser light scattering (MALLS) detector and an Optilab DSP differential refractometer, both from Wyatt Technology. Calculations were performed using the software package ASTRA from Wyatt Technology. The MALLS detector used a $30 \mathrm{~mW}, 690 \mathrm{~nm}$, linearly polarized gallium arsenide laser, and the differential refractometer used $690 \mathrm{~nm}$ light with a Wollaston prism. Tetrahydrofuran (THF) was used as the eluant at a flow rate of $1 \mathrm{~mL} \mathrm{~min}^{-1}$ and a temperature of $35^{\circ} \mathrm{C}$. No calibration standards were used and $\mathrm{d} n / \mathrm{d} c$ values were obtained for each injection assuming $100 \%$ mass elution from the columns and assuming dilute conditions where the second virial coefficient is negligible.

The second system used a Waters 410 differential refractometer with two Polymer Laboratories $30 \mathrm{~cm}$ long PLgel $10 \mu \mathrm{m}$ analytical columns connected in series. These columns were calibrated with monodisperse polystyrene samples and the polydispersity index (PDI) was measured using THF flowing at a rate of $0.9 \mathrm{~mL} \mathrm{~min}^{-1}$ as the mobile phase.

The last system consisted of a $30 \mathrm{~cm}$ long Polymer Laboratories PLgel $10 \mu \mathrm{m}$ preparative column connected to the same Waters 410 differential refractometer. This column was only used to fractionate and remove high molecular weight cross-linked polymer byproduct from the ABA LC polymers and was not calibrated.

\section{Phase transition temperature determination}

The transition temperatures and phases of the SGLCPs were determined using both a Zeiss polarized optical microscope (POM) with a Mettler FP82 hot stage and a Perkin Elmer DSC7 differential scanning calorimeter (DSC) using the Pyris software. In the microscope the temperature was slowly raised at between 1 and $5{ }^{\circ} \mathrm{C} \mathrm{min}^{-1}$ and the phases were identified along with the temperature at which phase transitions began. In the DSC method the samples were heated well into the isotropic phase to remove any thermal history. Then the temperature was raised at a rate of $10{ }^{\circ} \mathrm{C} \min ^{-1}$ and Perkin Elmer's Pyris computer software (version 3.04) was used to determine the onset temperature of the various phase transitions. The DSC was calibrated using indium and zinc as standards at a heating rate of $10{ }^{\circ} \mathrm{C} \mathrm{min}-1$.

\section{Nuclear magnetic resonance (NMR)}

${ }^{1} \mathrm{H}$ NMR was performed on a Mercury-Vx $300 \mathrm{MHz}$ NMR spectrometer with the software package VNMR Version 6.1B using 32 scans with a $1 \mathrm{~s}$ delay time. Experiments were run at room temperature using $\mathrm{CDCl} 3$ as a solvent with 10 to $20 \mathrm{mg}$ of polymer per $\mathrm{mL}$.

\section{Rheometry}

Rheometry was performed on a Rheometrics Fluids Spectrometer (RFS II) or a TA Instruments ARES-RFS rheometer equipped with a $50 \mathrm{~mm}$ or $25 \mathrm{~mm}$ diameter titanium shear cell in either a parallel-plate or a cone-and-plate geometry having a 0.0202 radian cone angle. No surface treatment was applied and the samples were heated into the isotropic state prior to measurement to erase any thermal history.

\section{Synthesis of ABA block-copolymer}

A "polymer analogous" approach to synthesis allowed a model series of high molecular weight polymers to be created with different mesogenic side groups but the same degree of polymerization. A single poly[styrene-block-(1,2-butadiene)block-styrene] triblock copolymer (ABA) was used as the prepolymer for LC triblocks ABASiBB, ABASiCB5 and ABASiCB4 (Fig. 1). ABA was synthesized by anionic polymerization with sequential addition of styrene, butadiene, and styrene $^{15}$ and used as received from Polymer Source (Montreal, Quebec). The various mesogenic units were attached to the pendant vinyl groups of poly(1,2-butadiene) by hydrosilylation. ${ }^{16-18}$

\section{Synthesis of SiCB4 mesogen}

All reagents were used as received from Aldrich, unless otherwise noted. 4'-cyano-4-hydroxybiphenyl (CHB), purchased from TCI America, 95\%, was purified on a silica gel column using 33\% ethyl acetate in hexane as the mobile phase. The spacer was prepared by the reaction of 3-buten-1-ol (10 $\mathrm{mL}, 0.116 \mathrm{~mol})$ with one molar equivalent pyridine $(9.4 \mathrm{~mL}, 0.116 \mathrm{~mol})$ as a catalyst and two molar equivalents of tosyl chloride $(44.3 \mathrm{~g}, 0.232 \mathrm{~mol})$ in dichloromethane (DCM) $(150 \mathrm{~mL})^{19}$ (Fig. 2). Reagents were mixed at $0{ }^{\circ} \mathrm{C}$, but the reaction was allowed to run at room temperature for $10 \mathrm{~h}$. An excess of pyridine $(100 \mathrm{~mL})$ was added to form a salt with the remaining tosyl chloride and then combined with $400 \mathrm{~mL}$ of a $5 \%$ solution of $\mathrm{HCl}$ in water. The product, 3-butenyl-1tosylate, was removed by liquid-liquid extraction with DCM. Then trace amounts of tosyl chloride in the product were removed by liquid-liquid extraction with a fresh $5 \%$ solution of $\mathrm{HCl}$ in water. The organic layer was dried with anhydrous $\mathrm{MgSO}_{4}$ and the solvent was evaporated, giving an $83 \%$ yield $(21.8 \mathrm{~g}, 0.0963 \mathrm{~mol})$. The spacer was attached to CHB (11.19 g, 


\section{$5 \mathrm{CB}$}
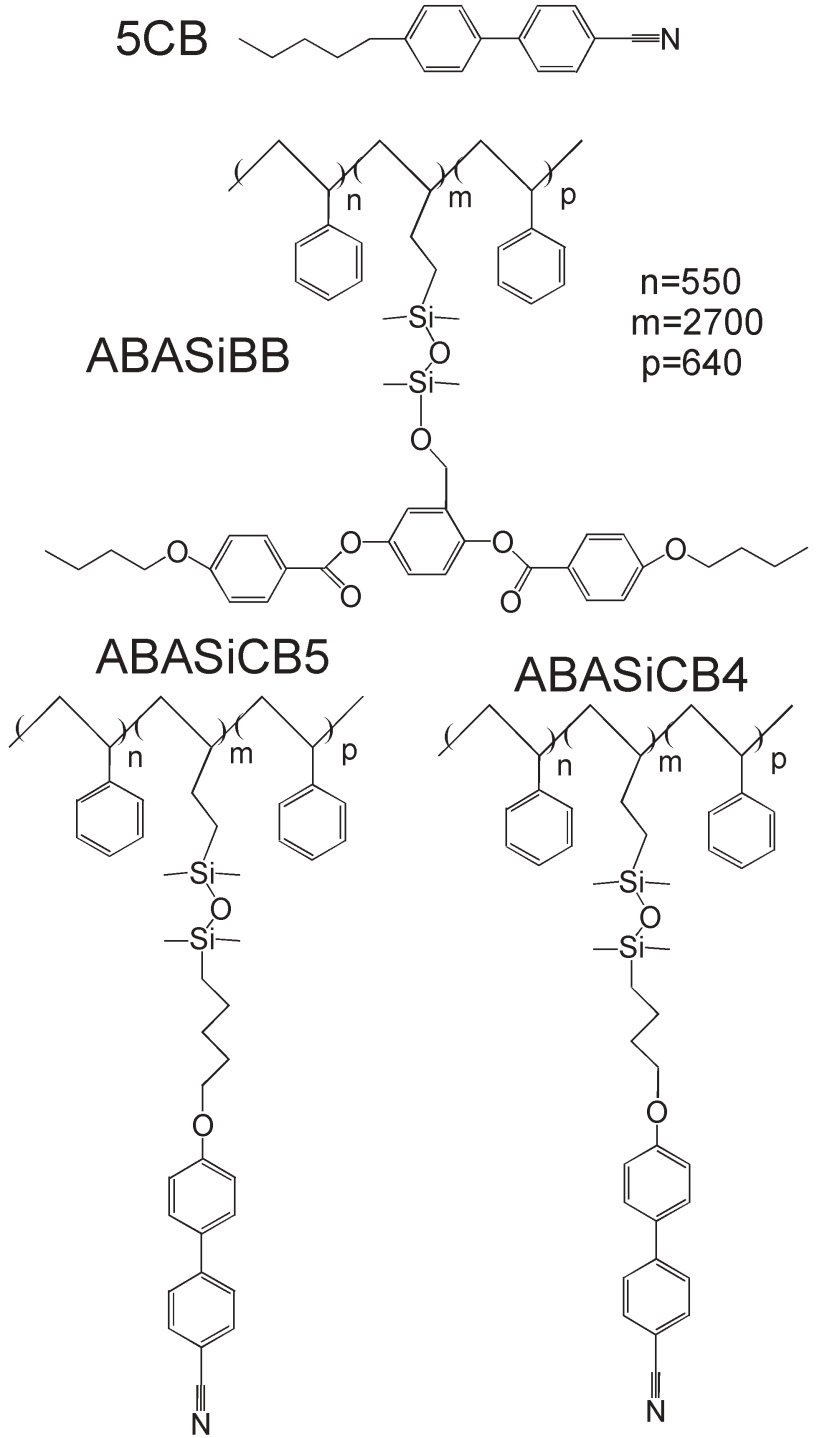

Fig. 1 Molecular structure of the nematic solvent 5CB and the ABA liquid crystalline polymers. These polymers were also made in a homopolymer form (without polystyrene endblocks) and named HSiBB, HSiCB5, and HSiCB4.

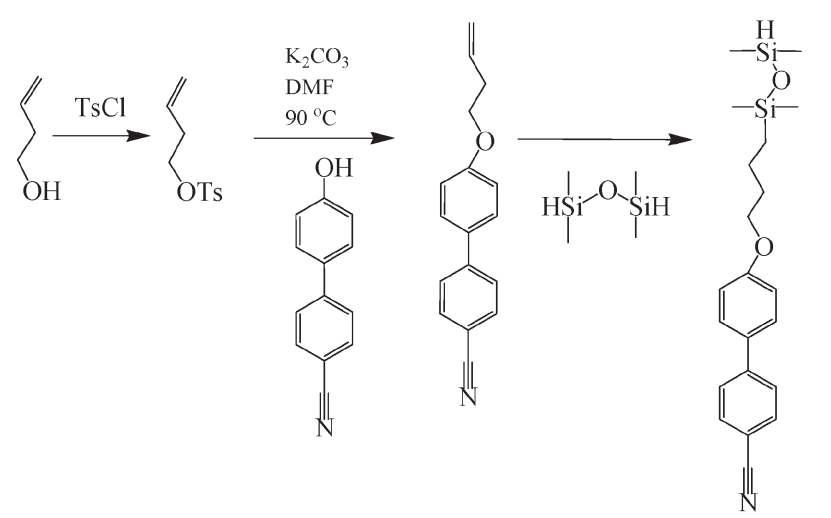

Fig. 2 Schematic for synthesis of SiCB4 mesogen.

$0.0573 \mathrm{~mol}$ ) using a seventy percent excess of 3-butenyl-1tosylate in $N, N$-dimethylformamide (DMF) $(50 \mathrm{~mL})$ and a seventy percent excess of anhydrous $\mathrm{K}_{2} \mathrm{CO}_{3}$ (13.3 g, $0.0962 \mathrm{~mol}$ ) at a temperature of $90{ }^{\circ} \mathrm{C}$ for $8 \mathrm{~h}$. The tosic acid by-product was precipitated by the addition of DCM, and the product 4-cyano-4'-(3-buteneoxy)-biphenyl (CBV4) was purified on a silica gel column using $10 \%$ ethyl acetate in hexane as the mobile phase. Finally, CBV4 was recrystallized from hexane to achieve $43 \%$ recovery $(6.14 \mathrm{~g}, 0.0246 \mathrm{~mol})$.

Ten molar equivalents of 1,1,3,3-tetramethyl disiloxane (TMDS) $(21.4 \mathrm{~mL}, 0.120 \mathrm{~mol})$ were combined with CBV4 $(3.00 \mathrm{~g}, 0.0120 \mathrm{~mol})$ in anhydrous toluene $(20 \mathrm{~mL})$ along with a few drops of the catalyst PC085, platinum-cyclovinylmethylsiloxane complex, or PC072, platinum-divinyltetramethyldisiloxane complex in xylene, both of which were purchased from United Chemical Technology. This reaction ran overnight at room temperature producing 4-cyano-4'-(5-(1,1,3,3tetramethyldisiloxane)butoxy)biphenyl (SiCB4).

Even a small amount of disiloxane byproduct can completely cross-link a high molecular weight polymer, so the SiCB4 must be highly purified prior to attachment to the poly(1,2-butadiene) backbone. The excess TMDS, bp $71{ }^{\circ} \mathrm{C}$, and toluene, bp $110{ }^{\circ} \mathrm{C}$, were removed by boiling under vacuum at $80{ }^{\circ} \mathrm{C}$ resulting in a product that should have been completely free of TMDS. Any trace amounts of water in the system have the potential to form 1,1,3,3,5,5,7,7-octamethyltetrasiloxane (OMTS), which would have a significantly higher boiling point than TMDS and consequently not be removed by this process. Therefore, a silica gel column was used to remove this and other byproducts of the reaction. The column was dried by blowing argon through the column while heating it with a propane torch and then anhydrous hexane was added to make a slurry and set up the column. The mesogen was added to the column in an anhydrous solution of toluene and hexane, and a generous amount of anhydrous hexane was run through the column until the mesogen nearly stopped moving, as seen with a UV lamp, removing the OMTS. Since the mesogen does not travel far with hexane, an anhydrous solution of 5\% ethyl acetate in hexane was used to move it down the column and separate it from other byproducts. When necessary, this column purification was repeated until the product SiCB4 was colorless.

\section{Synthesis of SiCB5 mesogen}

A $30 \%$ excess of 5-bromopentene $(7.89 \mathrm{~mL}, 0.0666 \mathrm{~mol})$ in DMF (100 mL) was combined with CHB (10 g, $0.0512 \mathrm{~mol})$ and one molar equivalent anhydrous $\mathrm{K}_{2} \mathrm{CO}_{3} \quad(7.08 \mathrm{~g}$, $0.0512 \mathrm{~mol})$ and allowed to react at a temperature of $90{ }^{\circ} \mathrm{C}$ for $3 \mathrm{~h}$ (Fig. 3). The product 4-cyano-4'-(3-penteneoxy)biphenyl, CBV5, was then purified on a silica gel column using $5 \%$ ethylacetate in hexane as the mobile phase with a final yield of $85 \%$ (11.5 g, $0.0435 \mathrm{~mol})$. The attachment of TMDS to yield 4-cyano-4' -(5-(1,1,3,3-tetramethyldisiloxane)pentoxy)biphenyl (SiCB5) and its subsequent purification was identical to that previously described for SiCB4.

\section{Synthesis of SiBB mesogen}

The synthesis of this mesogenic unit was similar to that used by researchers making polyacrylates..$^{20,21}$ 4-Butoxybenzoic acid (10 g, $0.0515 \mathrm{~mol}$ ) was converted into 4-butoxybenzoyl 


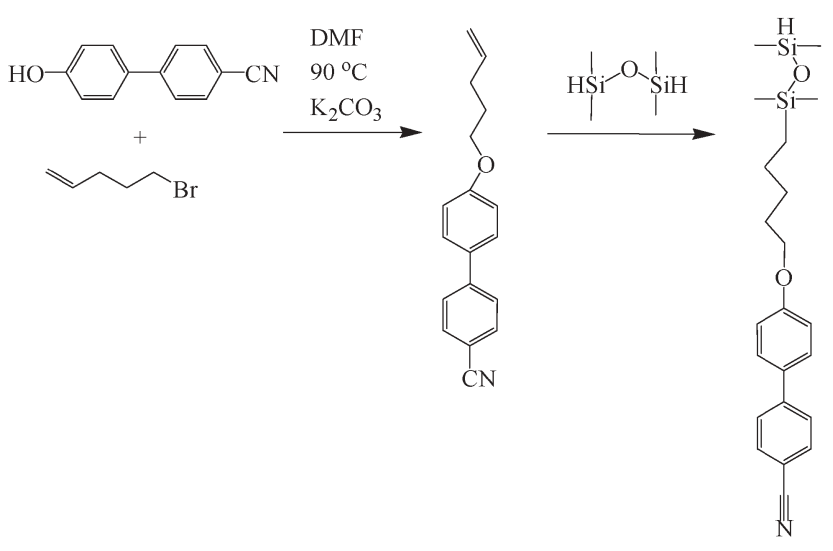

Fig. 3 Schematic for synthesis of SiCB5 mesogen.

chloride using a large excess of $\mathrm{SOCl}_{2}(20 \mathrm{~mL}, 0.275 \mathrm{~mol})$ as the solvent at $60{ }^{\circ} \mathrm{C}$ for one hour (Fig. 4). Excess $\mathrm{SOCl}_{2}$ was removed by evaporation under vacuum at $60{ }^{\circ} \mathrm{C}$. 4-butoxybenzoyl chloride was added at $10 \%$ excess to 2,5-dihydroxybenzaldehyde (3.2 g, $0.0198 \mathrm{~mol})$ (Lancaster Chemical) in a DCM $(30 \mathrm{~mL})$ solution with pyridine $(10 \mathrm{~mL})$, and the reaction was allowed to proceed at room temperature for several hours. The product 2,5-di(4-butoxybenzoate)-benzaldehyde was purified by liquid-liquid extraction using DCM and a $1 \mathrm{~N}$ solution of $\mathrm{HCl}$ (to remove excess pyridine) followed by another extraction using DCM and an saturated aqueous solution of $\mathrm{NaHCO}_{3}$ (to remove benzoic acid). The product was further purified by recrystallization from $85 \%$ ethanol in water $(100 \mathrm{~mL})$.

The spacer was attached to the aldehyde using Wilkinson's Catalyst, $^{22}$ (chlorotris(triphenylphospine)-rhodium(I), $40 \mathrm{mg}$ ), with ten molar equivalents TMDS $(35.1 \mathrm{~mL}, 0.198 \mathrm{~mol})$ in toluene $(100 \mathrm{~mL})$. The reaction was allowed to progress for $40 \mathrm{~min}$ at $60{ }^{\circ} \mathrm{C}$. The product 1,4-bis(4-butoxybenzoate)-2methyl[(1,1,3,3-tetramethyl-disiloxane)oxy]-benzene (SiBB) was purified on a silica gel column in a manner similar to that used for SiCB4 producing an overall yield of $50 \%$.

\section{Attachment of mesogen to ABA backbone}

The synthetic methods for using 1,1,3,3-tetramethylydisiloxane (TMDS) to attach a mesogenic group to a polymer backbone

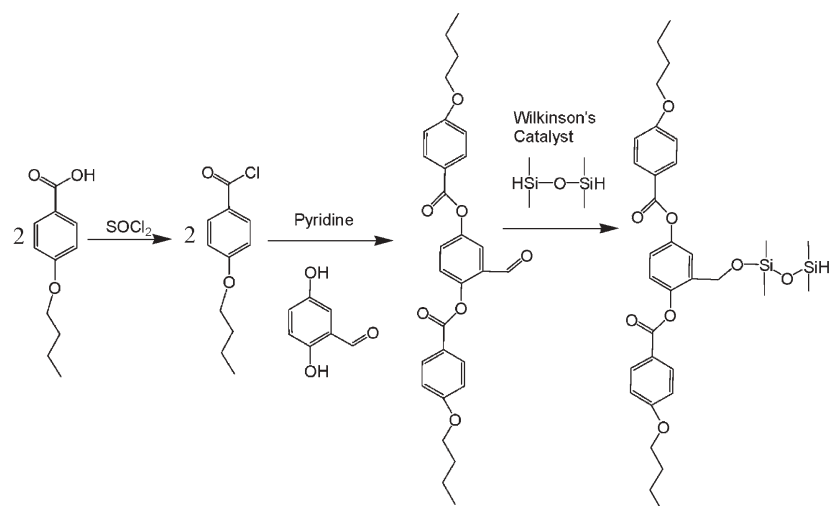

Fig. 4 Schematic for synthesis of SiBB mesogen. were similar to the work by other researchers ${ }^{16,17,22,23}$ but the relatively high molar mass of our polymers required more effort to purify our products. To attach the side groups, ABA prepolymer (1 g, 0.0088 mol vinyl groups) was dissolved in anhydrous THF $(40 \mathrm{~mL})$ and combined with three molar equivalents for SiCB4 and $\mathrm{SiCB} 5$ or five molar equivalents for SiBB (e.g. $10.1 \mathrm{~g} \mathrm{SiCB} 4,0.0264 \mathrm{~mol}$ ) of purified mesogens with an attached silane. A few drops of platinum catalyst PC085 was added and the mixture was stirred at $50{ }^{\circ} \mathrm{C}$ for a period of five to seven days (Fig. 5). A small sample was taken periodically and proton NMR was performed to monitor the reaction progress by the disappearance of the vinyl peak near $\Delta=5.5 \mathrm{ppm}$ (Fig. 6). When the size of the vinyl peak did not change significantly over two consecutive readings, the reaction was quenched by adding $5 \mathrm{~mL}$ of styrene and a fresh drop of platinum catalyst followed by stirring for one more day at $50{ }^{\circ} \mathrm{C}$ to remove any remaining silane. The product was then precipitated from solution by the addition of methanol (5 mL) and initially purified by repeatedly dissolving in THF and precipitating with methanol (Fig. 1). Further purification was accomplished using a preparative GPC column in THF solution to yield the final products. The polymer was then stored with $\sim 1 \%$ inhibitor, octadecyl-3-(3,5-di-tert-butyl-4-hydroxphenyl)propionate.

Regarding the stability of the polymer, some broadening of the molecular weight distribution occurred the first day after the polymer was precipitated out of solution. After that point, the polymer was stable for 6 months to a year when stored at room temperature in a drawer.

\section{Mixing of nematic gels}

To create homogeneous mixtures with $5 \mathrm{CB}$, the purified polymers were dissolved together with $5 \mathrm{CB}$ in DCM and the solvent subsequently removed by blowing air over the mixture until it became milky white. It was then placed in a vacuum at room temperature for one to two days. To be sure the polymer and $5 \mathrm{CB}$ were homogeneously mixed, the solution

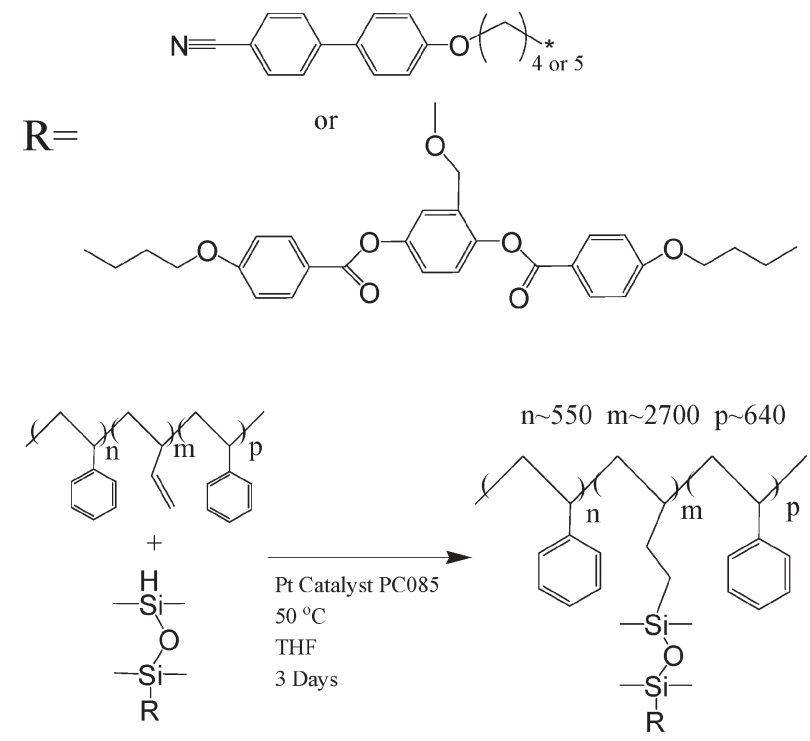

Fig. 5 Schematic for attachment of mesogens to a polymer backbone. 

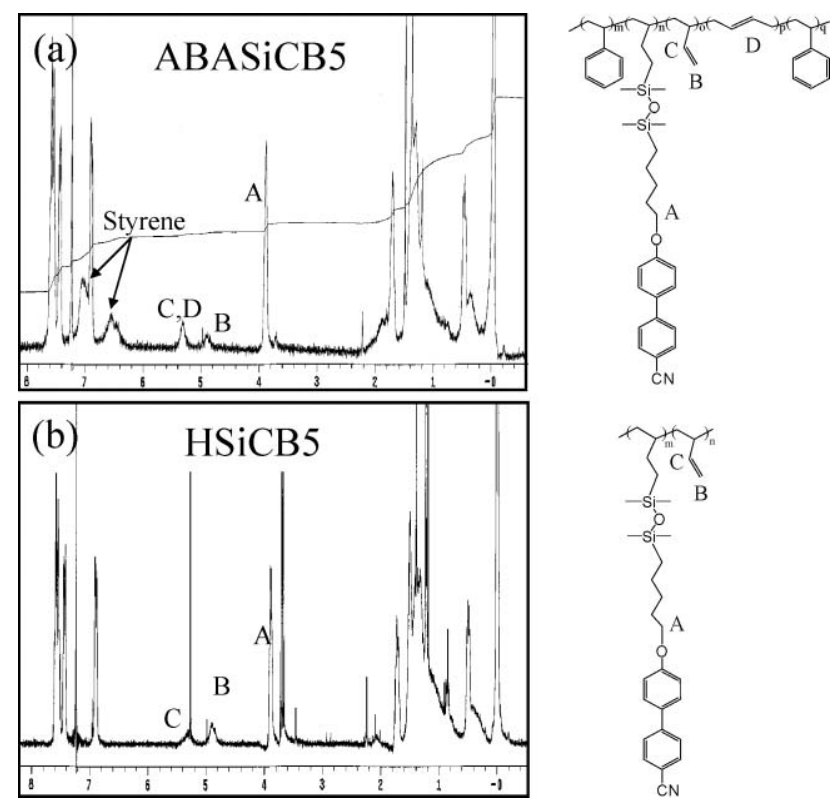

Fig. 6 NMR spectrum of (a) ABASiCB5 and (b) HSICB5 used to calculate the fractional attachment of mesogen to the pendant vinyl groups. The mole fraction of the components of the LC block can be calculated from the peak areas $A, B$, and $C$ as: $f_{\text {mesogen }}=(2 A) /(2 A+B+$ $2 C), f_{1,2}=(2 B) /(2 A+B+2 C)$ and $f_{1,4}=(2 C-B) /(2 A+B+2 C)$.

was periodically heated well into the isotropic phase (approximately $45{ }^{\circ} \mathrm{C}$ ) and stirred periodically during the drying process.

\section{Results}

\section{Polymer characteristics}

Three different mesogens were selected to confer solubility in 5CB (Fig. 1). Cyanobiphenyl-based SiCB4 and SiCB5 were chosen because matching the structure of the side-groups and LC solvent is known to increase solubility. ${ }^{24}$ Similarly, SiBB was chosen because the side-group has been shown to dissolve in mixtures of cyanobiphenyl-based LCs. ${ }^{21}$ The molecular weight of the final LC copolymer was calculated from the fractional attachment of mesogens onto the ABA prepolymer. This was found by comparing the integrated area for the ${ }^{1} \mathrm{H}$ NMR peaks corresponding to the alkene at 4.9 and $5.3 \mathrm{ppm}$ with the peak at $3.9 \mathrm{ppm}$ corresponding to a methylene group next to an ether link (Fig. 6). In SiCB4 and SiCB5 this methylene unit was next to the cyanobiphenyl and in $\mathrm{SiBB}$ it was next to the benzoate. Comparison of the areas of the two peaks of the vinyl groups allowed the determination of the relative number of 1,4-and 1,2-repeat units allowing us to verify that, within the experimental uncertainty, all the vinyl groups had been accounted for. Typically we found that between 87 and $94 \%$ of the available vinyl groups had reacted for $\mathrm{ABASiCB} 4$ and $\mathrm{ABASiCB} 5$, but for $\mathrm{ABASiBB}$ we were only able to achieve 70 to $80 \%$ conversion of the available vinyl groups.

The number average molecular weights, $M_{\mathrm{n}}$, of the different blocks of the ABA prepolymer were determined by Polymer Source using a MALLS system. They determined that the polystyrene endblocks had $M_{\mathrm{n}}$ equal to 57 and $67 \mathrm{~kg} \mathrm{~mol}^{-1}$ and the polybutadiene midblock had $M_{\mathrm{n}}$ equal to $146 \mathrm{~kg} \mathrm{~mol}^{-1}$, for a total molecular mass of $270 \mathrm{~kg} \mathrm{~mol}^{-1}$. After being converted into the final SGLCP, $M_{\mathrm{n}}$ of the midblock increased to between 1,100 and $1,350 \mathrm{~kg} \mathrm{~mol}^{-1}$ depending on the degree of attachment and the size of the side groups (Table 1). This reduces the fractional PS from $\sim 50 \%$ in the prepolymer to $\sim 10 \%$ in the LC triblock copolymer. The PDI of the LC triblocks is quite narrow for having such a high molar mass SGLCP domain. Nevertheless, its PDI was consistently higher than that of the ABA prepolymer (Table 1), which may be due to minute cross-linking during the attachment of side groups. A significant attribute of our synthetic approach is that the polymer is only involved in one reaction step, so these crosslinking reactions are kept to a minimum.

\section{Liquid crystal phase identification}

The isotropic transition temperatures of the bulk block copolymers ranged from 40 to $74{ }^{\circ} \mathrm{C}$ (Table 1). The type of $\mathrm{LC}$ order below the isotropization point was inferred from the

Table 1 Polymer characterization

\begin{tabular}{|c|c|c|c|c|c|c|c|}
\hline \multirow[b]{2}{*}{${ }^{a}$ Polymer } & \multirow[b]{2}{*}{$\begin{array}{l}{ }^{b} \text { Total } \\
M_{\mathrm{n}} / \mathrm{kg} \mathrm{mol}^{-1}\end{array}$} & \multirow[b]{2}{*}{ PDI } & \multirow[b]{2}{*}{$\begin{array}{l}\text { LC block } \\
M_{\mathrm{n}} / \mathrm{kg} \mathrm{mol}^{-1}\end{array}$} & \multicolumn{3}{|c|}{${ }^{c}$ LC block composition } & \multirow[b]{2}{*}{${ }^{e} T_{\mathrm{NI}} /{ }^{\circ} \mathrm{C}$} \\
\hline & & & & $\begin{array}{l}1,2 \text { Content } \\
(\mathrm{mol} \%)\end{array}$ & $\begin{array}{l}1,4 \text { Content } \\
(\mathrm{mol} \%)\end{array}$ & $\begin{array}{l}\text { Mesogen } \\
\text { content }\left(\mathrm{mol}^{2} \%\right)\end{array}$ & \\
\hline $\mathrm{H}$ & 63 & 1.04 & N/A & $\sim 98$ & $\sim 2$ & N/A & N/A \\
\hline HSiBB & 743 & 1.10 & 743 & 4 & $\sim 2$ & 94 & 120 \\
\hline HSiCB5 & 497 & 1.12 & 497 & 5 & $\sim 2$ & 93 & 70 \\
\hline HSiCB4 & 467 & 1.10 & 467 & 6 & $\sim 2$ & 92 & 60 \\
\hline${ }^{d} \mathrm{ABA}$ & 270 & 1.26 & N/A & 88 & 12 & N/A & N/A \\
\hline ABASiBB & 1341 & 1.4 & 1217 & 17 & 12 & 71 & 74 \\
\hline ABASiCB5 & 1172 & 1.4 & 1048 & 4 & 12 & 84 & 64 \\
\hline ABASiCB4 & 1134 & 1.4 & 1010 & 4 & 12 & 84 & 40 \\
\hline
\end{tabular}

${ }^{a}$ The polymer names starting with ABA were made from poly[styrene-block-(1,2-butadiene)-block-styrene] triblock copolymer. The polymer names starting with $\mathrm{H}$ were made with 1,2-polybutadiene. Polymers whose name ends with CB5 or CB4 have a cyanobiphenyl mesogen with a 4 or 5 methylene spacer connecting the siloxane group to the mesogen, respectively. Polymers ending with BB have the side-on mesogenic unit (Fig. 1). ${ }^{b}$ The molar masses of the butadiene pre-polymers were measured using MALLS but the final polymer molar masses listed were calculated based on the fractional conversion of the vinyl groups. ${ }^{c}$ The percents are based on the number of monomers in the LC block only.

${ }^{d}$ For the ABA triblock copolymers, the two styrene endblocks had molar masses of 57 and $67 \mathrm{~kg}$ mol ${ }^{-1}$ and the initial $1,2-$ polybutadiene center block had a molar mass of $M_{\mathrm{n}}=146 \mathrm{~kg} \mathrm{~mol}^{-1}$. ${ }^{e}$ Nematic to isotropic transition temperature $\left(T_{\mathrm{NI}}\right)$ of polymers determined using a Zeiss polarized optical microscope (POM) equipped with a Mettler FP82 hot stage. 
textures they exhibited when observed using POM. ABASiCB4 and $\mathrm{ABASiBB}$ had textures characteristic of nematic LCs, while ABASiCB5 had a rough focal conic texture characteristic of a smectic LC. The presence of a smectic phase in the analogous homopolymer, HSiCB5, was confirmed by wideangle X-ray scattering. ${ }^{25}$ The type of LC order in each case is consistent with that of the homopolymer analog ${ }^{25}$ and with previously established trends for SGLCPs (smaller spacer lengths lead to nematic phases and longer spacer lengths lead to smectic phases). ${ }^{26}$ Similarly, side-on SGLCPs are almost always nematic due to the difficulty of forming smectic layers when both the mesogen and the backbone are parallel to the director. ${ }^{27}$ The triblocks consistently have reduced isotropization temperatures relative to their homopolymer analogs. This may be a consequence of the reduced percentage of 1,2 repeat units $(88 \%$ compared to $98 \%$ ) in the prepolymer; and of the resulting reduction in the percentage of mesogenic units in the SGLCP midblock $(71-84 \%)$ as compared to the SGLCP homopolymers (92-94\%).

In solution in $5 \mathrm{CB}$ some of the triblock copolymers exhibited biphasic behavior. Mixtures of $5 \mathrm{CB}$ with ABASiCB4 exhibited a single sharp transition between the nematic and isotropic phases at all concentrations examined with the nematic to isotropic transitions temperature $\left(T_{\mathrm{NI}}\right)$ being slightly depressed $\left(2{ }^{\circ} \mathrm{C}\right.$ or less) relative to pure $5 \mathrm{CB}$. In contrast, mixtures of $5 \mathrm{CB}$ with $\mathrm{ABASiCB} 5$ at concentrations of $18 \mathrm{wt} \%$ or less are biphasic from ambient temperature up to their clearing point with polymer-rich and polymer-poor nematic phases coexisting; at concentrations of $20 \mathrm{wt} \%$ or greater there was a single, sharp transition. The tendency to phase separate may be due to the smectic character of this polymer. ${ }^{28}$ From this point on we restrict ourselves to singlephase solutions of ABASiCB5 (concentration > $20 \mathrm{wt} \%$ ). Mixtures of $5 \mathrm{CB}$ with $\mathrm{ABASiBB}$ at concentrations less than $10 \mathrm{wt} \%$ exhibit a narrow biphasic window with isotropic and nematic phases coexisting over a temperature range of approximately $1{ }^{\circ} \mathrm{C}$ near the isotropization temperature (which is within $3{ }^{\circ} \mathrm{C}$ of the $T_{\mathrm{NI}}$ of pure $5 \mathrm{CB}$ ). ${ }^{29}$ At concentrations greater than $10 \mathrm{wt} \%$ ABASiBB exhibited a single sharp transition with $T_{\mathrm{NI}}$ substantially greater than that of pure $5 \mathrm{CB}$.

The solubility of the triblocks differs somewhat from their homopolymer analogs: HSiCB5 has greater solubility than ABASiCB5 in 5CB but, conversely, HSiBB was less soluble than ABASiBB. HSiCB4 and HSiCB5 were soluble in $5 \mathrm{CB}$ in all proportions tested and exhibited a single sharp transition between the isotropic and nematic phases. The biphasic region of HSiBB solutions was observed to extend over a $10{ }^{\circ} \mathrm{C}$ range, much more pronounced than that of ABASiBB solutions.

\section{Dynamic mechanical analysis}

Rheometry was performed on single-phase solutions of the triblock copolymers in $5 \mathrm{CB}$ at various concentrations. As is often the case for block copolymer solutions, the viscoelastic relaxation spectrum changes shape with temperature, rather than simply shifting to faster timescales with increasing temperature. Their thermorheological complexity is particularly evident at temperatures near the isotropic-nematic transition (e.g., Fig. 7). A dramatic change in $G^{\prime}$ and $G^{\prime \prime}$ is observed between $T_{\mathrm{NI}}$ and $T_{\mathrm{NI}}+2{ }^{\circ} \mathrm{C}$. This abrupt change cannot be attributed to a change in the overall mobility of the solvent. The dominant viscosity in the nematic phase, $\eta_{1}$, is only approximately 3 to 6 times higher than the isotropic viscosity. $^{30}$

The enhancement of the modulus in the nematic phase correlates with strong aggregation of the PS endblocks evident in small-angle neutron scattering (SANS). ${ }^{31}$ Therefore, the increases in $G^{\prime}$ and $G^{\prime \prime}$ near $T_{\mathrm{NI}}$ can be attributed to microphase separation of the PS endblocks and the formation of a physical network at high enough polymer concentrations. With increasing concentration, a plateau in $G^{\prime}$ emerges in the nematic phase: it is not present at $1 \mathrm{wt} \%$ and is clearly evident at 3 and $5 \mathrm{wt}^{\%}$, indicating gelation (Fig. 8). At all concentrations there is a dense spectrum of relaxation times: At $T<T_{\mathrm{NI}}$ there is no minimum in $G^{\prime \prime}$ for any of the concentrations examined, even when there is a broad plateau in $G^{\prime}$, in contrast to the single relaxation time "Maxwell model" that approximately describes some telechelic associative polymer gels. ${ }^{32}$ At $1 \mathrm{wt}^{\%} \%$ ABASiBB (Fig. 8a) the relaxation spectrum resembles power law behavior with an exponent of $2 / 3$, i.e. $\left(G^{\prime}=3^{1 / 2} \cdot G^{\prime \prime} \sim \omega^{2 / 3}\right)$. For 3 and $5 \mathrm{wt} \%$ ABASiBB, as the temperature increases toward the isotropization temperature $\left(T_{\mathrm{I}}\right)$, the plateau modulus decreases (Fig. 8b,c). Terminal behavior is observed in the isotropic phase.

The block copolymer with end-on attachment of the mesogens to the SGLCP midblock (ABASiCB4) at $3 \mathrm{wt}^{\%} \%$ in 5CB has dynamic moduli (Fig. 7a) similar to those of the $3 \mathrm{wt} \%$ solution of $\mathrm{ABASiBB}$, which has side-on attachment (Fig. 8b). In the nematic phase, both materials have storage moduli that fall weakly with decreasing frequency with a slope that steepens as $T$ increases to $T_{\mathrm{I}}$, and loss moduli that do not have a minimum (dense spectrum of relaxation times). In the isotropic phase at $T>T_{\mathrm{NI}}+1{ }^{\circ} \mathrm{C}$, both systems show terminal behavior. Just above the phase transition, $T_{\mathrm{NI}}<T<T_{\mathrm{NI}}+1{ }^{\circ} \mathrm{C}$ (not available for $3 \mathrm{wt} \% \mathrm{ABASiBB}$ ), the isotropic solution has significantly slower dynamics than at slightly higher temperatures. The persistence of slow relaxation processes is also evident for the $5 \mathrm{wt} \%$ solutions of ABASiBB and $\mathrm{ABASiCB} 4$ and is prominent in the $20 \mathrm{wt} \%$ solution of ABASiCB5.

Restricting our attention to single-phase solutions of the end-on type polymer with longer spacer, ABASiCB5 was only examined at a concentration of $20 \mathrm{wt} \%$. Its dynamic moduli are an order of magnitude greater than those observed in the more dilute gels of ABASiBB and ABASiCB4 (Fig. 9). Similar to nematic gels of ABASiBB and ABASiCB4, a nematic gel of $20 \mathrm{wt} \%$ ABASiCB5 in 5CB shows $G^{\prime}$ gradually decreasing as frequency decreases and $G^{\prime \prime}$ with no local minimum. The longest relaxation time progressively drops by three orders of magnitude as the temperature increases from $T_{\mathrm{NI}}$ to $T_{\mathrm{NI}}+$ $50{ }^{\circ} \mathrm{C}$. Furthermore, thermorheological complexity clearly persists for at least $30^{\circ} \mathrm{C}$ above $T_{\mathrm{NI}}$; the slopes of $G^{\prime}$ and $G^{\prime \prime}$ at intermediate frequency increase with increasing temperature.

Data that span a wide temperature range can often be viewed on a reduced frequency scale, $a_{\mathrm{T}} \omega$, chosen to offset that portion of the temperature dependence that is attributed to the change in solvent viscosity. Such shifting for materials that undergo a phase change has received much criticism because 

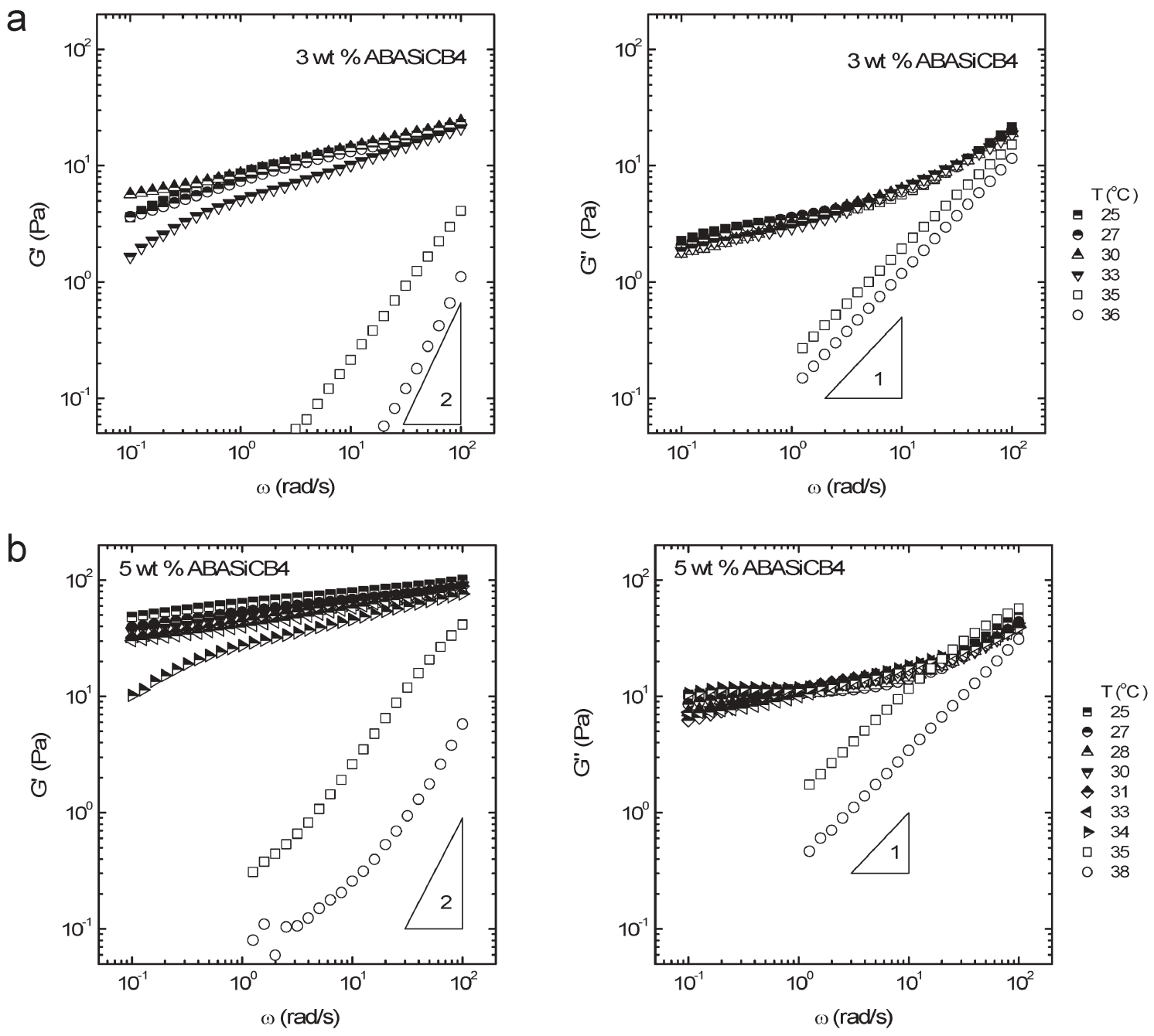

Fig. 7 Dynamic storage modulus $\left(G^{\prime}\right)$ and loss modulus $\left(G^{\prime \prime}\right)$ of a mixture of (a) $3 \mathrm{wt} \%$ and (b) $5 \mathrm{wt} \%$ of the nematic end-on polymer ABASiCB4 in $5 \mathrm{CB}$. The mixture is isotropic for all $T \geqslant 35^{\circ} \mathrm{C}$ (empty symbols) and nematic for $T \leqslant 34{ }^{\circ} \mathrm{C}$ (half-filled symbols).

they should not (and generally do not) obey time-temperature superposition. When the temperature dependence of viscosity of $5 \mathrm{CB}$ is applied to our data, the resulting shifts are mild. The small shifts bring the data at high frequencies closer together and all of the noteworthy features cited above remain.

\section{Discussion}

Associative polymers for LC gels are a recently developed class of materials that provide sensitive and rapid electro-optic response, and open routes to large-area display fabrication. ${ }^{11}$ The gels can be shear aligned into an orientation parallel to either the shear direction or the velocity gradient direction, depending on whether the polymer mesogens are attached side-on (ABASiBB) or end-on (ABASiCB4, ABASiCB5), respectively. The polymer network preserves the "field-off" alignment state, yet is sufficiently dilute to permit rapid reorientation dynamics. An important characteristic of these gels is their reversible sol-gel transition, particularly with temperature. The rheology of the gels reported here reveals the molecular basis for switching from the sol to gel state.
A unique feature of triblock copolymer gels in LC solvent is the discontinuous change in solvent quality coincident with the first-order LC phase transitions. ${ }^{31}$ This phenomenon is responsible for the large increase in $G^{\prime}$, as much as an order of magnitude, observed over a $\sim 1{ }^{\circ} \mathrm{C}$ decrease from $T_{\mathrm{NI}}$ to $T_{\mathrm{NI}}-1{ }^{\circ} \mathrm{C}$ in dilute (greater than $1 \mathrm{wt} \%$ and up to $5 \mathrm{wt} \%$ ) triblock copolymer gels; the solubility of the PS endblocks is much greater in the isotropic phase than in the nematic. The transition from gel to sol takes place over an extremely small temperature range compared to bulk $\mathrm{BCPs}$ or physical gels of BCPs swollen with isotropic solvent. The transition is broadened in bulk BCPs due to transient compositional fluctuations, ${ }^{33}$ and in isotropic gels due to the continuous change in solvent quality with temperature. ${ }^{34,35}$ The enormous sensitivity of LC gels to temperature and polymer concentration allows mechanical properties to be tuned with small changes to these parameters; the elastic modulus of ABASiCB4 gels, for example, increases by an order of magnitude when polymer concentration is increased from 3 to $5 \mathrm{wt} \%$ and decreases drastically when temperature is raised from 33 to $35^{\circ} \mathrm{C}$. 
a

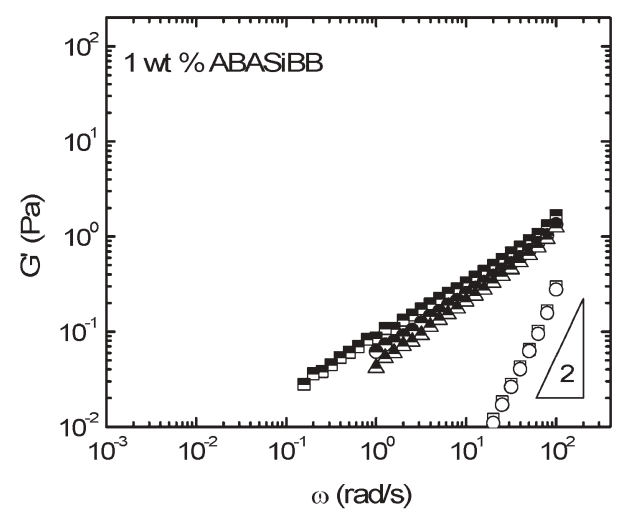

b

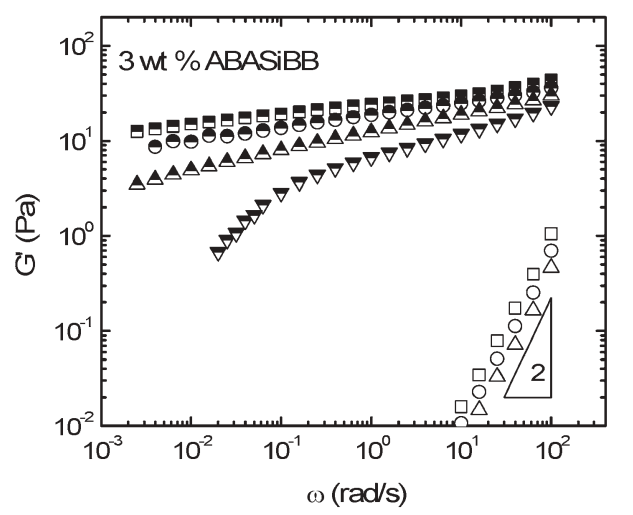

C

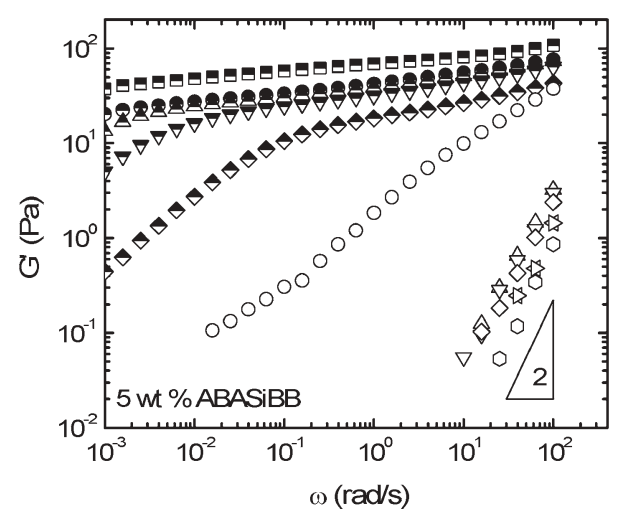

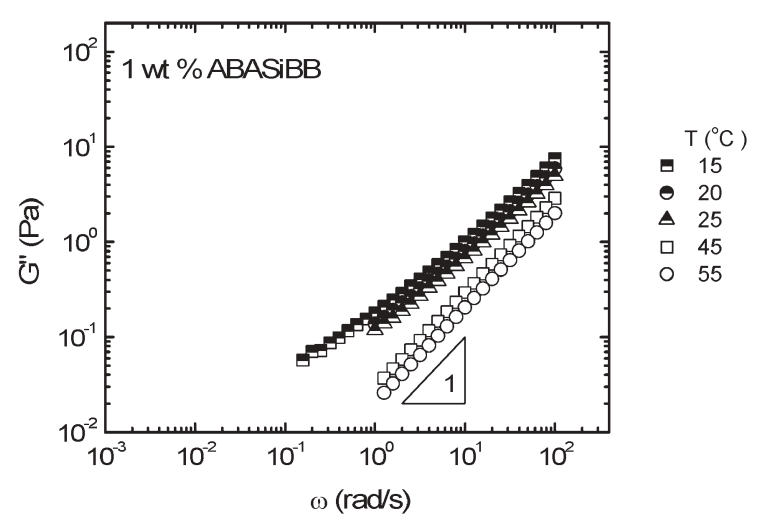
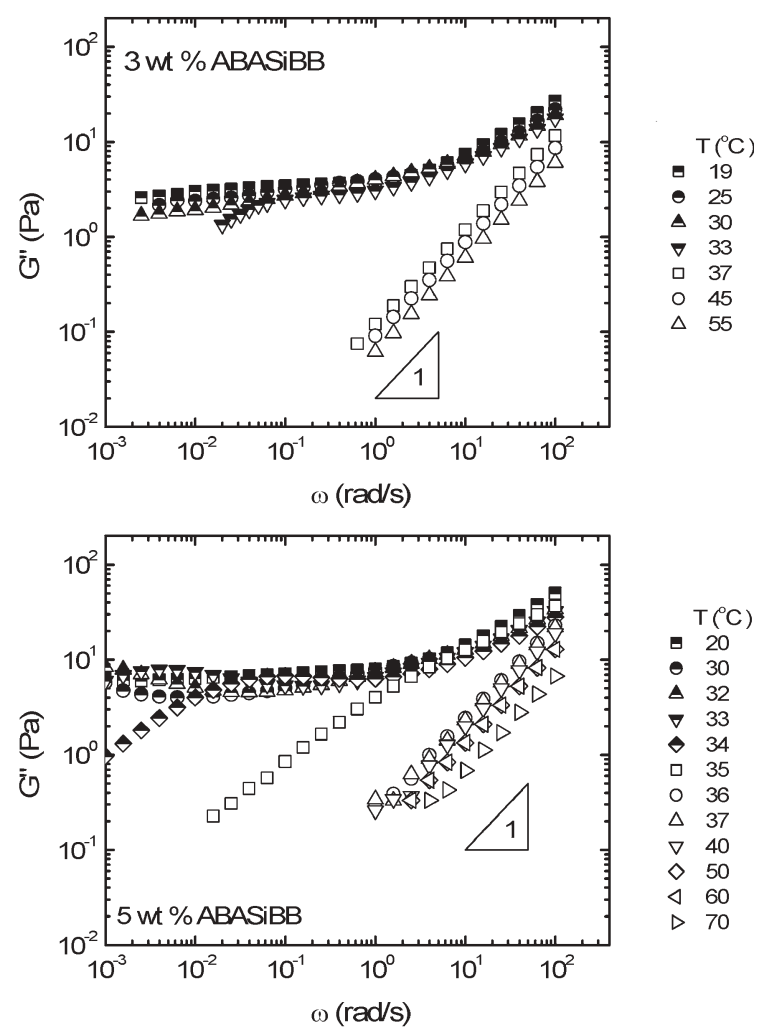

Fig. 8 Dynamic storage modulus $\left(G^{\prime}\right)$ and loss modulus $\left(G^{\prime \prime}\right)$ of a mixture of (a) $1 \mathrm{wt} \%$ (b) $3 \mathrm{wt} \%$ and (c) $5 \mathrm{wt} \%$ of the nematic side-on polymer ABASiBB in 5CB. The mixture is isotropic for all $T \geqslant 35{ }^{\circ} \mathrm{C}$ (open symbols) and nematic for $T \leqslant 34{ }^{\circ} \mathrm{C}$ (half-filled symbols).

Rheology of the LC gels also shows an unexpected thermodynamic driving force for their self-assembly. Thermorheological complexity in the isotropic phase of the $20 \mathrm{wt} \%$ ABASiCB5 gel is evidence that PS endblock association persists above $T_{\mathrm{NI}}$. We did not expect this because PS homopolymer of a comparable concentration and molecular weight to the PS endblocks is soluble in isotropic 5CB. ${ }^{36}$ This led us to investigate the phase behavior of solutions of PS homopolymer and SGLCP homopolymer having side-group structure identical to ABASiCB4. Their ternary phase behavior revealed that the differing affinity of isotropic $5 \mathrm{CB}$ for the PS and SGLCP resulted in macroscopic separation into PS-rich and SGLCP-rich phases, even under conditions where each homopolymer would be miscible with isotropic $5 \mathrm{CB} .{ }^{31}$ The reduced solubility of PS upon addition of SGLCP gives insight into the behavior of the block copolymer gels: PS endblocks associate even in isotropic 5CB because the SGLCP midblock drives the PS endblock out of the SGLCP-5CB solution.

The roughly parallel decline of $G^{\prime}$ and $G^{\prime \prime}$ with decreasing frequency is unusual when compared to bulk BCPs or isotropic BCP gels. Whereas the endblock exchange time is often observed to be the dominant relaxation process in endassociative polymer gels, ${ }^{32}$ our data show this not to be the case; though it may be the longest relaxation time there are many other processes of comparable importance with relaxation times spread throughout the spectrum. This property of the gels may have implications for the network-induced memory effect that serves to preserve the LC molecules' alignment state.

The exceptional electro-optic and mechano-optic responsiveness of these gels coupled with their unique thermal 

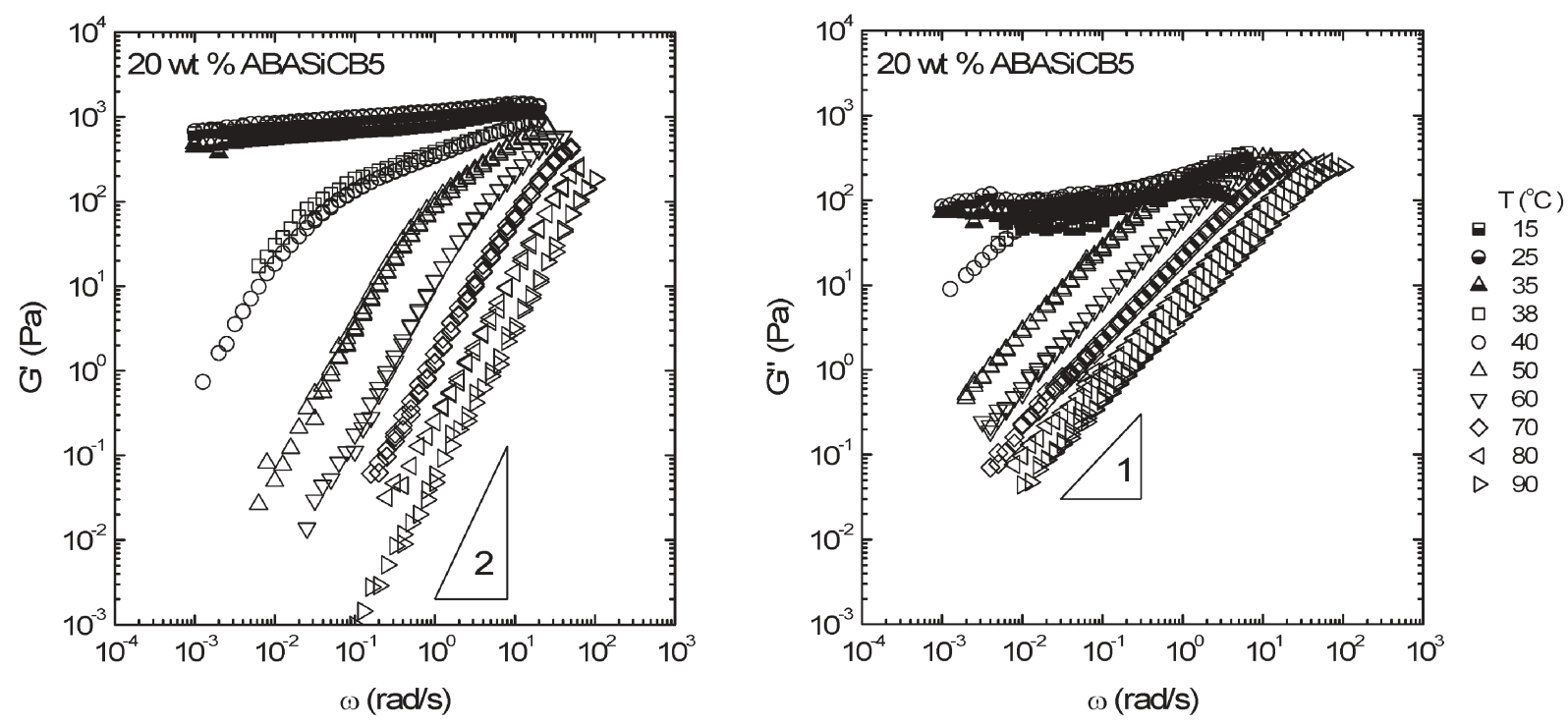

Fig. 9 Dynamic storage modulus $\left(G^{\prime}\right)$ and loss modulus $\left(G^{\prime \prime}\right)$ of a mixture of $20 \mathrm{wt} \%$ of the smectic end-on polymer ABASiCB5 in $5 \mathrm{CB}$. The mixture is isotropic for all $T \geqslant 38^{\circ} \mathrm{C}$ (empty symbols) and nematic for $T \leqslant 35^{\circ} \mathrm{C}$ (half-filled symbols).

sensitivity suggests they would be ideal candidates for use in large-area printable display technology. A solution of triblock copolymer in LC solvent could be heated just above $T_{\mathrm{NI}}$ to transform the material into a liquid amenable to roll-to-roll or ink-jet printing. Gelation will spontaneously occur subsequent to printing by simply allowing the solution to cool to room temperature, and the desired alignment state can be achieved by mechanical shear. Alternatively, these block copolymers could be utilized as rheology modifiers to facilitate large-area coating of LC materials; adding as little as $1 \mathrm{wt} \%$ polymer can significantly enhance the viscosity of the LC without adversely affecting the rate of formation of a desired director configuration.

\section{Conclusions}

The network structure of self-assembled LC gels is thermoreversible. Swelling a well-defined, high molecular weight coilSGLCP-coil block copolymer with a small-molecule LC solvent produces nematic gels with a physically cross-linked, macroscopic polymer network. Because of the abrupt change in solvent quality at the first order phase transition from nematic to isotropic order, the physical network also changes abruptly at $T_{\mathrm{NI}}$. Three different SGLCP midblocks flanked by PS endblocks all show that when the polymer concentration is dilute in $5 \mathrm{CB}$, the polystyrene endblocks are well dissolved in the isotropic state, but microphase separate upon cooling into the nematic state. Thus, at low concentration, gelation is turned "on" and "off" by crossing $T_{\mathrm{NI}}$ : the microphase separation temperature of the BCP coincides with the change in solvent quality. When the concentration of polymer is sufficiently high to raise the microphase separation temperature of the $\mathrm{BCP}$ above $T_{\mathrm{NI}}$, there is a sudden change in rheological properties at $T_{\mathrm{NI}}$ indicative of a transition from one segregated state to another. Dramatic changes in network structure are observed over small temperature windows (as little as $1{ }^{\circ} \mathrm{C}$ ) due to the sharp change in LC order near the isotropization point.

\section{Acknowledgements}

The authors acknowledge financial support from the AFOSR LC-MURI (f4962-97-1-0014), the ARCS foundation, the NSF Department of Materials Research (DMR-0216491), the NDSEG fellowship, and the James-Irvine Foundation. We would like to thank Steven Smith of Procter and Gamble for providing us with 1,2-polybutadiene samples, and María Lujan Auad for helpful discussions.

\section{References}

1 C. Park, J. Yoon and E. L. Thomas, Polymer, 2003, 44, 6725.

2 M. Muthukumar, C. K. Ober and E. L. Thomas, Science, 1997, 277, 1225.

3 J. Sanger, W. Gronski, H. Leist and U. Wiesner, Macromolecules, 1997, 30, 7621.

4 M. Anthamatten, J.-S. Wu and P. T. Hammond, Macromolecules, 2001, 34, 8574.

5 J. Sanger, W. Gronski, S. Maas, B. Stuhn and B. Heck, Macromolecules, 1997, 30, 6783.

6 M. Anthamatten, W. Y. Zheng and P. T. Hammond, Macromolecules, 1999, 32, 4838.

7 I. W. Hamley, V. Castelletto, Z. B. Lu, C. T. Imrie, T. Itoh and M. Al-Hussein, Macromolecules, 2004, 37, 4798.

8 W. Y. Zheng and P. T. Hammond, Macromolecules, 1998, 31, 711.

9 C. C. Honeker and E. L. Thomas, Chem. Mater., 1996, 8, 1702.

10 C. Osuji, P. J. Ferreira, G. Mao, C. K. Ober, J. B. V. Sande and E. L. Thomas, Macromolecules, 2004, 37, 9903.

11 M. D. Kempe, N. R. Scruggs, R. Verduzco, J. Lal and J. A. Kornfield, Nat. Mater., 2004, 3, 177.

12 R. Verduzco, G. Meng, J. A. Kornfield and R. B. Meyer, Phys. Rev. Lett., 2006, accepted for publication.

13 T. Mansare, R. Decressain, C. Gors and V. K. Dolganov, Mol. Cryst. Liq. Cryst., 2002, 382, 97.

14 K. Skarp, S. T. Lagerwall and B. Stebler, Mol. Cryst. Liq. Cryst., 1980, 60, 215.

15 J. Sanger, C. Tefehne, R. Lay and W. Gronski, Polym. Bull. (Berlin), 1996, 36, 19. 
16 A. Moment, R. Miranda and P. T. Hammond, Macromol. Rapid Commun., 1998, 19, 573.

17 M. A. Hempenius, R. G. H. Lammertink and G. J. Vancso, Macromol. Rapid Commun., 1996, 17, 299.

18 I. Ojima, M. Nihonyanagi, T. Kogure, M. Kumagai, S. Horiuchi, K. Nakatsugawa and Y. Nagai, J. Organomet. Chem., 1975, 94, 449.

19 X. F. Ren, E. Turos, C. H. Lake and M. R. Churchill, J. Org. Chem., 1995, 60, 6468.

20 Q.-F. Zhou, A.-M. Li and X.-D. Feng, Macromolecules, 1987, 20, 233.

21 M. C. Chang, H. W. Chiu, X. Y. Wang, T. Kyu, N. Leroux, S. Campbell and L. C. Chien, Liq. Cryst., 1998, 25, 733.

22 M. A. Hempenius, R. G. H. Lammertink and G. J. Vancso, Macromolecules, 1997, 30, 266.

23 A. Moment and P. T. Hammond, Polymer, 2001, 42, 6945

24 H. Kihara, R. Kishi, T. Miura, T. Kato and H. Ichijo, Polymer, 2001, 42, 1177.

25 M. L. Auad, M. D. Kempe, J. A. Kornfield, S. Rendon, W. R. Burghardt and K. Yoon, Macromolecules, 2005, 38, 6946.

26 V. P. Shibaev, S. G. Konstromin and N. A. Plate, Eur. Polym. J., 1982, 18, 651.
27 S. Lecommandoux, M. F. Achard, F. Hardouin, A. Brulet and J. P. Cotton, Liq. Cryst., 1997, 22, 549 .

28 J. D. Laffitte, M. F. Achard, F. Hardouin, H. T. Nguyen and G. Sigaud, Liq. Cryst., 1994, 17, 487.

29 In a previous publication, ref. 10, we reported mixtures of ABASiBB with $5 \mathrm{CB}$ to be single-phase for all concentrations between $0.5-20 \mathrm{wt} \%$. At that time we had not observed the narrow biphasic window described here.

30 H. Kneppe, F. Schneider and N. K. Sharma, Ber. Bunsenges. Phys. Chem., 1981, 85, 784.

31 N. R. Scruggs, J. A. Kornfield and J. Lal, Submitted for Publication, 2006

32 T. Annable, R. Buscall, R. Ettelaie and D. Whittlestone, J. Rheol., 1993, 37, 695.

33 G. H. Fredrickson and F. S. Bates, Annu. Rev. Mater. Sci., 1996, 26, 501.

34 K. Inomata, D. Nakanishi, A. Banno, E. Nakanishi, Y. Abe, R. Kurihara, K. Fujimoto and T. Nose, Polymer, 2003, 44, 5303.

35 T. Sato, H. Watanabe and K. Osaki, Macromolecules, 2000, 33, 1686.

36 H. Hori, O. Urakawa and K. Adachi, Macromolecules, 2004, 37, 1583.

\section{Find a SOLUTION with books from the RSC}

\section{Choose from exciting textbooks, research level books or reference books in a wide range of subject areas, including:}

- Biological science

- Food and nutrition

- Materials and nanoscience

- Analytical and environmental sciences

- Organic, inorganic and physical chemistry

\section{Look out for 3 new series coming soon ...}

- RSC Nanoscience \& Nanotechnology Series

- Issues in Toxicology

- RSC Biomolecular Sciences Series

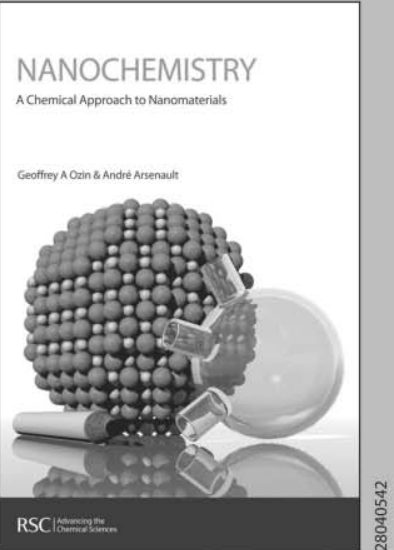

www.rsc.org/books 